\title{
46,XY/45,X mosaicism in an amniotic fluid cell culture: suppression of abnormal cell line after subcultivation
}

\author{
LIS HASHOLT \\ From the Institute of Medical Genetics, University of Copenhagen, Tagensvej 14, Copenhagen, Denmark
}

SUMmARY An abnormal cell population, 45,X, appeared in 3 of 4 cell lines established from an amniotic fluid specimen obtained from a normal mid-trimester pregnancy. Two of the cell lines were subjected to repeated chromosome analyses until VII passage. The abnormal cells were suppressed after repeated trypsinisations; simultaneously, fibroblast-like cells outgrew the cultures, which were previously predominated by epithelial-like cells. Polyploidy was found in 0 to $12 \%$ of the cells, the highest level existing in the early passages. The question of whether chromosomally abnormal cells present in primary cultures and the early subcultures reflect the karyotype of the fetus is discussed.

Polyploidy is regularly found among cultured amniotic fluid cells. More recently, chromosomal mosaicisms have been reported in a number of cases without confirmation of the abnormal chromosome constitution in cells from the fetus or from the live born child. A few cases of true mosaicism have also been published. A survey of the mosaic findings has been published by Hsu et al. (1976). Later publications confirm that pseudomosaicism is occasionally found (Aula et al., 1978, Rodriguez et al., 1978). The diagnostic dilemma is obvious. Firstly, is the mosaic constitution of the amniotic fluid cells representative of the karyotype of the fetus, or is it an anomaly that arises in vivo or in vitro without any consequences for the child? Secondly, if the mosaicism found reflects the condition of the fetus, how would it influence the phenotype of the child? This paper, in which a case of $46, X Y / 45, X$ mosaicism is reported, deals with the first problem.

In order to obtain a rapid diagnosis, primary cultures or second to third passage cultures are generally used for chromosome analysis. In the present amniotic fluid specimen, repeated chromosome analyses were performed, and the karyotype of the cells was followed until the seventh passage. Usually the primary cultures have a predominance of epithelial-like cells which have a short life span and disappear after a few trypsinisations; subsequently other cell types predominate in the cultures. If the aberrant cells of a mosaic amniotic fluid cell culture

Received for publication 26 September 1978 are also lost after a few passages, it might be possible to relate the chromosome abnormality to cell type. The consequences with respect to the cytogenetic diagnosis are discussed.

\section{Materials and methods}

The amniotic fluid specimen was obtained by transvaginal amniocentesis from a second trimester pregnancy before induced abortion. The fluid was part of normal material analysed with respect to the effect of culture conditions on the behaviour of amniotic fluid cell cultures; therefore, a chromosome analysis was not made on the fetus.

The following day, the amniotic fluid was centrifuged for $10 \mathrm{~min}$ at $260 \mathrm{~g}$, and the cells were resuspended in $6 \mathrm{ml}$ medium composed of Eagle's MEM with Hank's salt solution supplemented with $20 \%$ fetal calf serum and glutamine. Penicillin and streptomycin were added as antibiotic agents. Four cell lines were started by division of the cell suspension into four Leighton tubes with cover slips. After the first trypsinisation, the cells were transferred to $30 \mathrm{ml}$ plastic flasks (Nunc, Denmark) with a growth surface area of $25 \mathrm{~cm}^{2}$. The living cells were examined with an inverted phase contrast microscope (Leitz-Diavert). Cultures were harvested for chromosome analysis after trypsinisation. The slides were stained with the conventional Giemsa technique and with the quinacrine fluorescence technique (Q-banding). 
Results

Two cell populations, $46, \mathrm{XY}$ and $45, \mathrm{X}$, were found in cultures from the amniotic fluid. Most of the cells had a normal karyotype but the 45, X chromosome constitution was found in cells from three of the four cell lines. The $\mathrm{Y}$ chromosome, when present, had a normal bright fluorescence. The cytogenetic findings are summarised in the Table. All the cells analysed from line 3, III passage had a $\mathrm{Y}$ chromosome. Thirty mitoses from cell line 1 were analysed in V passage; only one of these was 45,X. Cell line 4 was karyotyped in II, V, VI, and VII passage. The abnormal 45, X cells amounted to 8 of 70 cells analysed from passage II. During the following passages, the aberrant cell population decreased. One of 19 cells from $V$ passage was lacking the $\mathrm{Y}$ chromosome. Sixty metaphases were analysed from passage VI and 95 metaphases from passage VII, but no cells with the aberration were found among them. The 45,X cells were most frequently found among cells from cell line 2, passage III, but here, too, the abnormal cell population was reduced after subcultivations, as only 2 of 69 cells in passage VII had this karyotype.

Chromosomal aberrations of different types appeared sporadically in all the lines. Thus, a new clone with the chromosome constitution $46, X Y$, $-D,+G$ emerged in cell line 2 from the III to VI passage.

The proportion of polyploid cells in each passage is stated for about 200 cells when possible. Generally 0 to $3 \%$ of the cells showed polyploidy, but in two cultures the frequency was as high as $12 \%$.

The morphology of the cells, epithelial-like or fibroblast-like, was scored before harvest of the cells. The term fibroblast-like includes cells with a real fibroblast-shape as well as AF-cells, as they have been defined by Hoehn et al. (1974). The epitheliallike cells predominated only in the very early stages of growth; after III passage the fibroblast-like cells overgrew the cultures.

\section{Discussion}

The presence of an aberrant cell clone in amniot fluid cell cultures can be explained in several ways: (1) The anomaly arose in vivo, as a result of irregular mitoses in the fetus, in the amnion, or in the ce露 exfoliated to the amniotic fluid. (2) The anomaf arose in vitro, as a result of culture conditions. The latter hypothesis is excluded if the clone is observet in cultures from successive amniocenteses of the sange pregnancy, or if the clone, as in the present case, is found in cultures from different cell lines, or 昆 different primary cultures. With respect to the firs? hypothesis, the exfoliated cells do not seem to divid as no mitoses were observed among uncultured cells prepared immediately after amniocentesis, and 18 cells were found to be labelled by autoradiographig examination after incubation for $4 \mathrm{~h}$ with ${ }^{3} \mathrm{P}$ thymidine (T. S. Clausen, 1978, unpublished datad. Similarly, Hoehn et al. (1974) found no labelled cells among $2 \times 10^{-3}$ cells examined after incuba tion for $24 \mathrm{~h}$ with ${ }^{3} \mathrm{H}$ thymidine and medium. Sô, the cell division which gave rise to the abnormळil clone would have taken place in the amnion or in the fetus itself, a fact which does not help us to makecorrect chromosomal diagnosis of the child. In this connection, repeated amniocentesis is not expecter to give any new information. This has been coffirmed by the observations published so far, for example by Milunsky and Atkins (1976), who fourif only cells with a normal karyotype from the secorid amniocentesis, whereas a 46,XY/47, XXY cot stitution of cells from the first amniocentesis was also found in different tissues from the newbog infant. On the other hand, Sutherland et al. (1975) described a case of mosaicism in two successive amniocenteses, but fetal fibroblasts showed ond normal karyotypes.

Cells belonging to a cell population with the abnormal karyotype 45,X were found in 3 of 4 ciel lines from the present amniotic fluid specimen. stated above, tissue from the fetus was not obtained;

Table Cytogenetic results from 4 cell lines originating from one amniotic fluid specimen

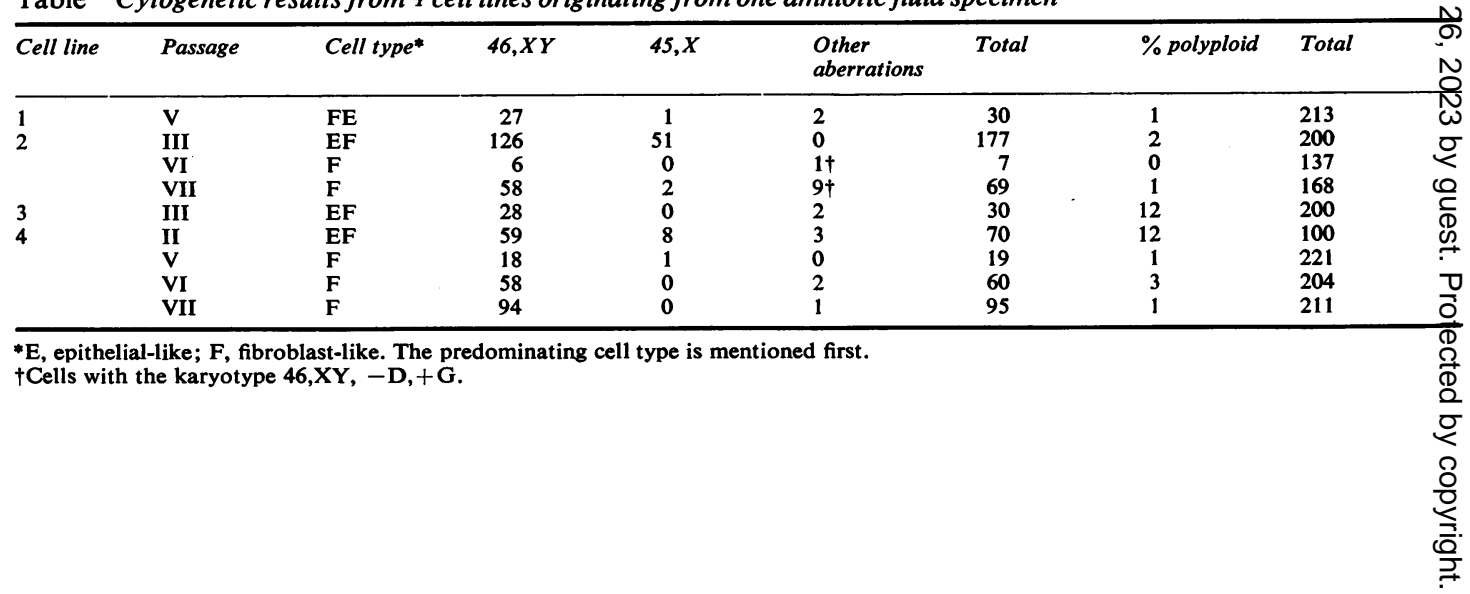


and the mosaic condition could not be verified. Mosaics with this karyotype have been described before in amniotic fluid cell cultures. Hsu et al. (1976) mentioned that 3 cases had been reported, including their own finding, where the anomaly was confirmed by culturing cells from the fetus. The mosaic conditions found at prenatal diagnosis generally include the same chromosome abnormalities seen frequently among liveborn infants, that is $45, \mathrm{X}$, monosomy $\mathrm{G}$, and trisomy $\mathrm{D}$ and $\mathrm{G}$. Therefore, discrimination between real mosaics and pseudomosaics is not possible from the type of aberration.

In the 2 cell lines where several passages were analysed, the $45, \mathrm{X}$ cell population was considerably diminished after repeated subcultivations. As chromosome preparations used for prenatal diagnosis are made on primary cultures or after one subcultivation, little is known of the fate of the abnormal cells in the mosaic cases published so far. Sutherland et al. (1975) karyotyped 5 cases of mosaicism in both the primary culture and a subculture originating from it after trypsinisation. In 2 instances, the chromosome analysis revealed abnormal cells only in the primary cultures. In the present experiment, the disappearance of the cells with epithelial-like morphology paralleled the disappearance of the chromosome abnormality. The epithelial-like cells are supposed to originate from various parts of the fetus, for example, the skin, the respiratory tract, the urogenital tract, and the amnion. Cultures from the amnion are composed of epithelial-like cells with a short lifespan; cell lines from this tissue are difficult to establish (Boué et al., 1975), and special culture conditions are required (Conover and Hirschhorn, 1971). The difficulty in establishing amnion cell cultures indicates that the origin of the fibroblast-like cells in amniotic fluid cell cultures is not the amnion, but the fetus itself, and consequently the chromosome constitution of these cells should be representative of the fetus.

A high incidence of polyploidy is frequently found in amniotic fluid cell cultures. The number of polyploid cells were recorded in all the cultures harvested for chromosome analysis; the results were ambiguous but the incidence tended towards a higher level in the first passages. A similar observation was made by Kohn and Robinson (1970), who found $13 \%$ polyploid metaphases after 20 days in culture, but no polyploid cells after 35 days. It is possible that this chromosome anomaly is also associated with the epithelial-like cells. Hahnemann (1974) found tetraploidy in up to $10 \%$ of cells from amnion cultures, but the frequency of polyploid cells in cultures from human fetal lung, skin, and kidney is at an equal level, from 0.5 to $9 \%$ (Earley et al., 1976). The origin of the polyploid cells in amniotic fluid cell cultures is thus unsolved.

If the epithelial-like cells show an unstable chromosome constitution, then it is inexpedient to prepare primary cultures for cytogenetic analysis, and the karyotyping would more usefully be carried out in a later passage on fibroblast-like cells. However, an extended culture time is unfortunate from a diagnostic point of view, and adds new problems to cytogenetic analysis, for instance, the selection of a clone or the emergence of new clones. Further investigations of the origin and the fate of the cell types found in the cultures will be necessary to clarify whether it is possible to discriminate between a real mosaic condition and pseudomosaicism.

I wish to thank Dr E. Niebuhr for his help with the fluorescence analyses, and Anja Boesen, Minna Jensen, and Elsebeth Lund Christensen for skilful technical assistance.

This study was supported in part by Carl og Ellen Hertz's Fond.

\section{References}

Aula, P., von Koskull, H. and Ryynänen, M. (1978). A case of $46, X X / 47, X X,+G$ mosaicism in amniotic fluid not confirmed in the fetus. Clinical Genetics, 13, 103.

Boué, A., Boué, J., Cure, S., Deluchat, C., and Perraudin, N. (1975). In vitro cultivation of cells from aneuploid human embryos. Initiation of cell lines and longevity of the cultures. In Vitro, 11, 409-413.

Conover, J. H., and Hirschhorn, K. (1971). Establishment and genetic stability of human amnion cell lines. Laboratory Investigation, 24, 38-40.

Earley, E. M., Finley, S. C., Finley, W. H., Hsu, L. Y. F., Kim, H. J., Petricciani, J. C., and Vinson, P. C. (1976). Karyology of primary human fetal cell cultures. In Vitro, 12, 639-641.

Hahnemann, N. (1974). Early prenatal diagnosis; a study of biopsy techniques and cell culturing from extraembryonic membranes. Clinical Genetics, 6, 294-306.

Hoehn, H., Bryant, E. M., Karp, L. E., and Martin, G. M. (1974). Cultivated cells from diagnostic amniocentesis in second trimester pregnancies. I. Clonal morphology and growth potential. Pediatric Research, 8, 746-754.

Hsu, L. Y. F., Hyon, J. K., Hausknecht, R., and Hirschhorn, K. (1976). Prenatal diagnosis of $45, X / 46, X Y$ mosaicism with postnatal confirmation in a phenotypically normal male infant. Clinical Genetics, 10, 232-238.

Kohn, G., and Robinson, A. (1970). Tetraploidy in cells cultured from amniotic fluid. Lancet, $1,778$.

Milunsky, A., and Atkins, L. (1976). Prenatal diagnosis of chromosomal mosaicism. Journal of Pediatrics, 88, 367-368.

Rodriguez, M. L., Luthy, D., Hall, J. G., Norwood, T. H., and Hoehn, H. (1978). Amniotic fluid cell mosaicism for presumptive trisomy 20. Clinical Genetics, 13, 164-168.

Sutherland, G. R., Bowser-Riley, S. M., and Bain, A. D. (1975). Chromosomal mosaicism in amniotic fluid cell cultures. Clinical Genetics, 7, 400-404.

Requests for reprints to Dr Lis Hasholt, Institute of Medical Genetics, University of Copenhagen, Tagensvej 14, DK-2200 Copenhagen N, Denmark. 